\title{
Session 5
}

\section{Grand minima and Historical Records}




\title{
Stars in magnetic grand minima: where are they and what are they like?
}

\author{
Steven H. Saar and Paola Testa \\ Smithsonian Astrophysical Observatory, \\ 60 Garden Street, Cambridge,MA 02138, USA \\ email: saar@cfa.harvard.edu, ptesta@cfa.harvard.edu
}

\begin{abstract}
We explore various ideas of what a star in a Maunder-like magnetic minimum would look like, and ways of finding stars in such a state, and make some estimates of their physical and magnetic activity properties. We discuss new X-ray observations of a small selection of candidates for being in magnetic grand minima. These are then compared with the Sun and other low activity stars.
\end{abstract}

Keywords. stars: magnetic fields, stars: late-type, stars: evolution, stars: activity, Sun: magnetic fields, Sun: activity

\section{Introduction}

Solar magnetic grand minima (=MGM), their properties and connection to normal cycle minima are clearly of interest for many reasons (hence, this conference!). Unfortunately, all recent examples of solar MGM fall outside the time horizon of modern instrumentation, so we are left with an imperfect record of sunspot counts and mostly indirect data of other types (e.g., cosmogenic isotopes). What is it about the solar dynamo that causes MGM? What are solar conditions like during these events? What governs the length of MGM and how does the Sun recover its cycle again? The existing data are useful, but more and better information would certainly help.

It has long been realized that solar-like stars may be very useful in better understanding solar activity and cycles (e.g., Wilson 1978). Carefully selected stars could inform not only what solar MGM conditions might have been like, but also how MGM conditions and frequency vary with stellar age, mass, and other properties. The difficulty lies in determining just what a star in an MGM should look like. Indeed, it has recently been suggested that there are very few solid MGM star candidates (Wright 2004), implying the Sun may be highly unusual for having these epochs of magnetic somnambulence! Is this view correct? This paper will review the history of various attempts to define MGM star candidates ( $\S 2)$, lay out a new attempt at defining MGM stars ( 33 ), explore their properties $(\S 4)$, and discuss implications for the solar MGM properties and MGM origins, and future directions $(\S 5)$.

\section{A bit of history}

Early in the study of solar-like activity, the possibility of finding solar-like stars in MGM was recognized. Baliunas \& Vaughan (1985), in their review of Ca II HK measurements in cool stars, noted, "because all other weak emission-line stars in this range $[0.72 \leqslant(\mathrm{~B}-\mathrm{V})$ $\leqslant 0.76$ ] do vary, the implication is that HD 10700 may be in an epoch of a virtual lull in chromospheric activity similar to the Maunder minimum." This star has been repeatedly mentioned as an MGM candidate, and is still often viewed as such (Judge et al. 2004). 
Baliunas \& Jastrow (1990) published an intriguing paper studying a mix of measurements of $S_{\mathrm{HK}}$, the Mount Wilson Ca II HK core-to-continuum ratio, over time, including four flat activity stars (with low $\left\langle S_{\mathrm{HK}}\right\rangle$ and variability $\sigma_{S}$ ). The sample was restricted to stars of solar-like color $(0.60 \leqslant \mathrm{~B}-\mathrm{V} \leqslant 0.76)$. A histogram of the $S_{\mathrm{HK}}$ values revealed a bimodal distribution, with $\approx 30 \%$ lying in a low $\left\langle S_{\mathrm{HK}}\right\rangle$ peak. They suggested this meant that solar-like stars spend $\approx 30 \%$ of the time in MGM. Hall \& Lockwood (2004), however, used a larger sample of low activity stars and more even time sampling and found no such bimodality.

Saar \& Baliunas (1992) found that about 10-15\% of the Mount Wilson survey stars were of the flat activity class, almost all of them concentrated in the F and G stars. These seemed to be the best MGM candidates, and Saar (1998) argued many of them might be in MGM, since their rotation rates were similar to cycling stars, and yet their activity levels were strongly dependent on rotation, suggestive of a turbulent (non cycling) dynamo (Bercik et al. 2005). The number of flat stars with known $P_{\text {rot }}$, however, was small, making the statistics less than impressive.

Using a large survey of southern hemisphere stars, Henry et al. (1996) studied the distribution of calibrated $R_{\mathrm{HK}}^{\prime}=\left(F_{\mathrm{HK}}-F_{\mathrm{phot}}\right) / F_{\mathrm{bol}}$ values, where $F_{\mathrm{HK}}$ is the raw calibrated HK core flux (computed from $S_{\mathrm{HK}}$ ), $F_{\text {phot }}$ is the photospheric component of the HK core flux, and $F_{\text {bol }}$ is the bolometric flux (Noyes et al. 1984). $R_{\mathrm{HK}}^{\prime}$ values in principle should permit better comparison of stars with different temperatures, since different backgrounds $F_{\text {phot }}$ and continuum fluxes are accounted for. Henry et al. found that $\approx 5-$ $10 \%$ of the their sample formed a distinct low activity group with $\log R_{\mathrm{HK}}^{\prime} \leqslant-5.1$, and identified these with MGM candidates.

Wright (2004) studied $R_{\mathrm{HK}}^{\prime}$ derived by Wright et al. (2004) from a large database of exoplanet search spectra, comparing $R_{\mathrm{HK}}^{\prime}$ with the star's magnitude separation $\Delta \mathrm{M}_{V}$ from an Hipparcos-defined main sequence. He found that almost without exception, stars with $\log R_{\mathrm{HK}}^{\prime} \leqslant-5.1$ had $\Delta \mathrm{M}_{V}>1$, and were thus not strictly solar-like, as they were either evolved (subgiants) or had strongly different metallicity, or both. He therefore questioned whether there were any true MGM candidates, at least with the restriction $\log R_{\mathrm{HK}}^{\prime} \leqslant-5.1$. Wright (2004) also presciently noted that there might be problems with the $R_{\mathrm{HK}}^{\prime}$ calibration related to unaccounted-for metallicity and gravity effects. We return to this below.

Giampapa et al. (2006) took a different approach and surveyed the roughly solar age $(\approx 4$ Gyr) cluster M67. They found about $17 \%$ of dwarfs in the color range $0.58 \leqslant \mathrm{~B}-\mathrm{V}$ $\leqslant 0.76$ had $\left\langle S_{\mathrm{HK}}\right\rangle$ below solar minimum levels, and identified these as MGM candidates. For a more restricted range of very solar-like stars $(0.63 \leqslant \mathrm{~B}-\mathrm{V} \leqslant 0.67), \approx 19 \%$ (4 of 21$)$ lay below solar minimum in Ca II HK. Jenkins et al. (2008) looked at a large sample of southern dwarfs, and again adopting $\log R_{\mathrm{HK}}^{\prime} \leqslant-5.1$ for a MGM candidate, found $1-3 \%$ might be in such a state.

So, estimates of the fractions of stars in MGM have been generally dropping since the first studies, but is the fraction near zero (Wright 2004) or small but non-negligible (e.g., Giampapa et al. 2006)? How unusual is the Sun for having MGM episodes?

\section{Constructing a new set of MGM candidate criteria}

Wright (2004) raised some important issues concerning the most commonly used Ca II HK activity index. He noted that there is both metallicity and gravity effects that are entwined in the $S_{\mathrm{HK}}$ index, which complicate defining the activity minimum. Indeed, the traditional calibration to convert $S_{\mathrm{HK}}$ to a normalized photosphere-subtracted flux 
ratio $R_{\mathrm{HK}}^{\prime}$ does not take gravity or metallicity into account. Others have complained recently that $R_{\mathrm{HK}}^{\prime}$ does not correlate well with age for older ( $>1$ Gyr or so) stars (Pace \& Pasquini 2004; Saffe et al. 2005); it is likely these calibration issues are partly to blame. Inspired by Wright (2004), Saar (2006) tried to remove the effect of gravity and metallicity effects on determining the minimum Ca II HK fluxes (and, hence, where to best search for MGM candidates). By matching stars in the Wright et al. (2004) HK database with the detailed spectroscopic modeling of Valenti \& Fischer (2005), he compared the $R_{\mathrm{HK}}^{\prime}$ values with accurate $T_{\text {eff }}$, metallicities $[\mathrm{M} / \mathrm{H}]$, gravities $g$, and $v \sin i$ (Saar 2006; Saar 2011). A fit to the main sequence seen in the log $g-T_{\text {eff }}$ plane (Fig. 1) gave $>500$ spectroscopically confirmed dwarfs independent of metallicity. Subgiants were then excluded, since their dynamos might well have stopped functioning due to their (evolution-driven) lower rotation rates. When the $R_{\mathrm{HK}}^{\prime}$ of the Wright et al. (2004) sample was plotted against the log metallicity $[\mathrm{M} / \mathrm{H}]$ relative to solar from Valenti \& Fischer (2005), a striking dependence of the minimum $R_{\mathrm{HK}}^{\prime}$ values in dwarfs could be seen (Fig 2; Saar 2006;Saar 2011). A rough boundary of the minimum can be sketched as log $R_{\mathrm{HK}}^{\prime}(\mathrm{min})=-5.125-0.213[\mathrm{M} / \mathrm{H}]$. Since even metal-poor halo dwarfs can have cycles (e.g., HD 103095; Baliunas et al. 1995) there seems no reason to exclude stars of arbitrary $[\mathrm{M} / \mathrm{H}]$ from being candidate MGM stars. Thus, we can expect MGM candidates to reside among the lowest activity stars anywhere along this boundary. The previous criterion of $\log R_{\mathrm{HK}}^{\prime} \leqslant-5.1$ for all $[\mathrm{M} / \mathrm{H}]$ would appear to be too restrictive, ruling out all but a few true dwarfs.

Very low average activity is a probably necessary but certainly not sufficient condition to be an MGM candidate. (The caveat "probably" is included because we shouldn't

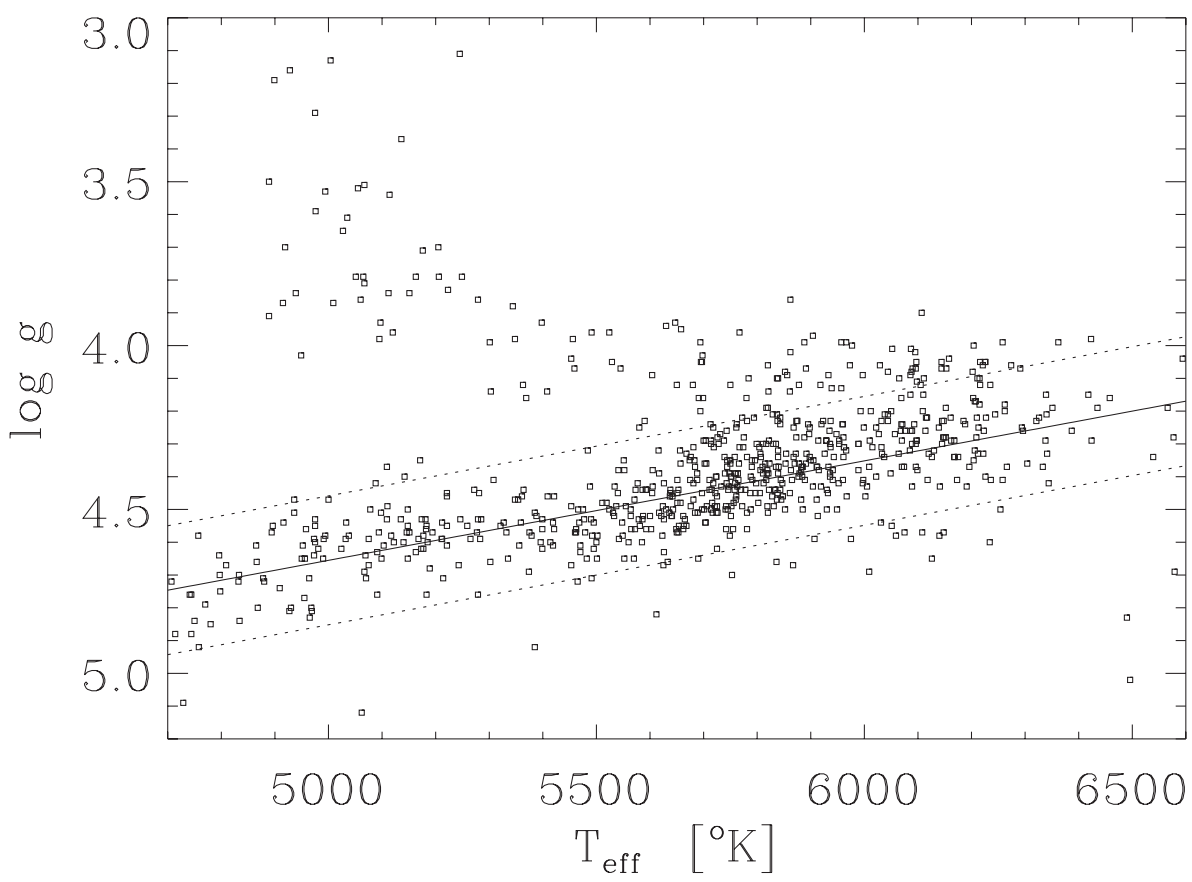

Figure 1. $T_{\text {eff }}$ vs. $\log g$ from Valenti \& Fischer (2005) for stars also in Wright et al. (2004). A linear fit to the main sequence (solid) and $2.3 \sigma_{\text {fit }}$ boundaries defining dwarfs (dotted) are shown (from Saar 2011). 


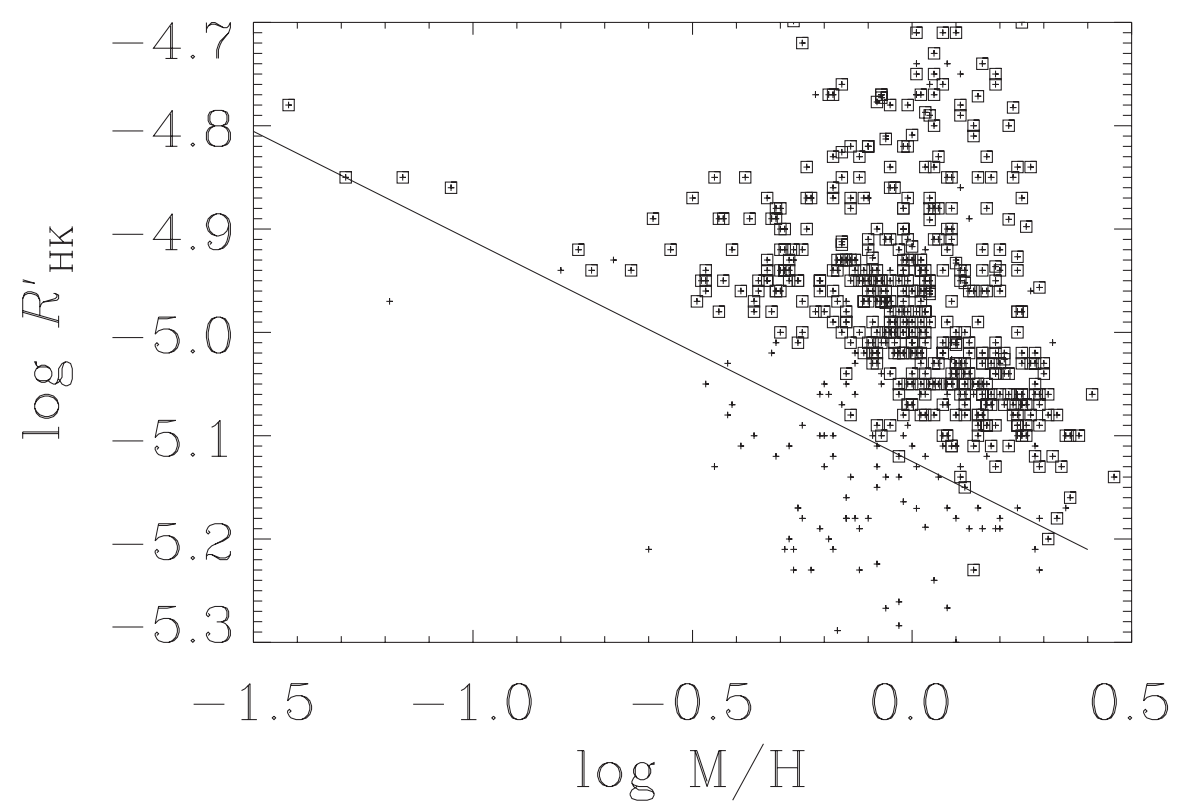

Figure 2. $R_{\mathrm{HK}}^{\prime}$ from Wright et al. (2004) vs. $\log \mathrm{M} / \mathrm{H}$ from Valenti \& Fischer (2005) for stars in common; dwarfs as defined by Fig. 1 are boxed. A linear lower boundary for $R_{\mathrm{HK}}^{\prime} \approx-0.213$ $[\mathrm{M} / \mathrm{H}]$ - 5.125, is drawn (solid); only one dwarf falls well below this boundary (from Saar 2011).

completely rule out the possibility that the activity level of an MGM state is related to the the mean activity level and/or rotation rate of the star when not in such a state. Faster rotators might have higher $\left\langle R_{\mathrm{HK}}^{\prime}\right\rangle$ in their grand minima than slower rotators.) A good MGM candidate should also have low HK variability and to have had sustained it for as long a time as possible. At a minimum, $\sigma_{S}$ should be derived from data which span an interval longer than the longest normal solar cycle minimum - about 4 years. Interestingly, just above the $R_{\mathrm{HK}}^{\prime}(\mathrm{min})$ boundary, for $\Delta \log R_{\mathrm{HK}}^{\prime}=\log R_{\mathrm{HK}}^{\prime}-\log R_{\mathrm{HK}}^{\prime}(\min )$ $\leqslant 0.054$, nearly all stars show $\sigma_{S} / S_{\mathrm{HK}} \leqslant 2 \%$ (Fig. 3). This is the variabiliy level Baliunas et al. (1995) used to define "flat activity" stars, and we adopt it again here. A $2 \% \mathrm{HK}$ variation criterion is a factor of $\sim 5$ below the Sun's over a typical cycle (as measured from the Sacramento Peak Observatory K line data over the last two cycles) and is $\sim 30 \%$ less than the average variation during the last three solar minima.

Our new MGM candidate critera are thus the following: (1) the star is a bona-fide dwarf as determined from spectroscopically determined $T_{\text {eff }}$ and gravity (Fig. 1); (2) The star shows $\log R_{\mathrm{HK}}^{\prime}<-5.125-0.213[\mathrm{M} / \mathrm{H}]+\delta$, where $[\mathrm{M} / \mathrm{H}]$ is the spectroscopically determined $\log$ metal abundance (relative to solar) and $\delta \approx 0.054$; and $(3) \sigma_{S} / S_{\mathrm{HK}} \leqslant 2 \%$ spanning at least $t_{\mathrm{obs}} \geqslant 4$ years of measurements (i.e., $>$ a solar minimum timescale).

Indirect evidence from cosmogenic isotopes such as ${ }^{10} \mathrm{Be}$ and ${ }^{14} \mathrm{C}$ indicates that cyclic modulation continued through the Maunder minimum (e.g., Usoskin et al. 2004), despite minimal spot modulation. It would appear that the magnetic cycle continued, producing weak activity (active network/plage and thus Ca II HK emission) without many spots. Thus, it seems reasonable to expect some HK variation during MGM, but this discussion underlines the uncertainties of the problem. Note also that, for example, the Dalton minimum was much shorter and less deep than the Maunder! Clearly, solar grand minima 


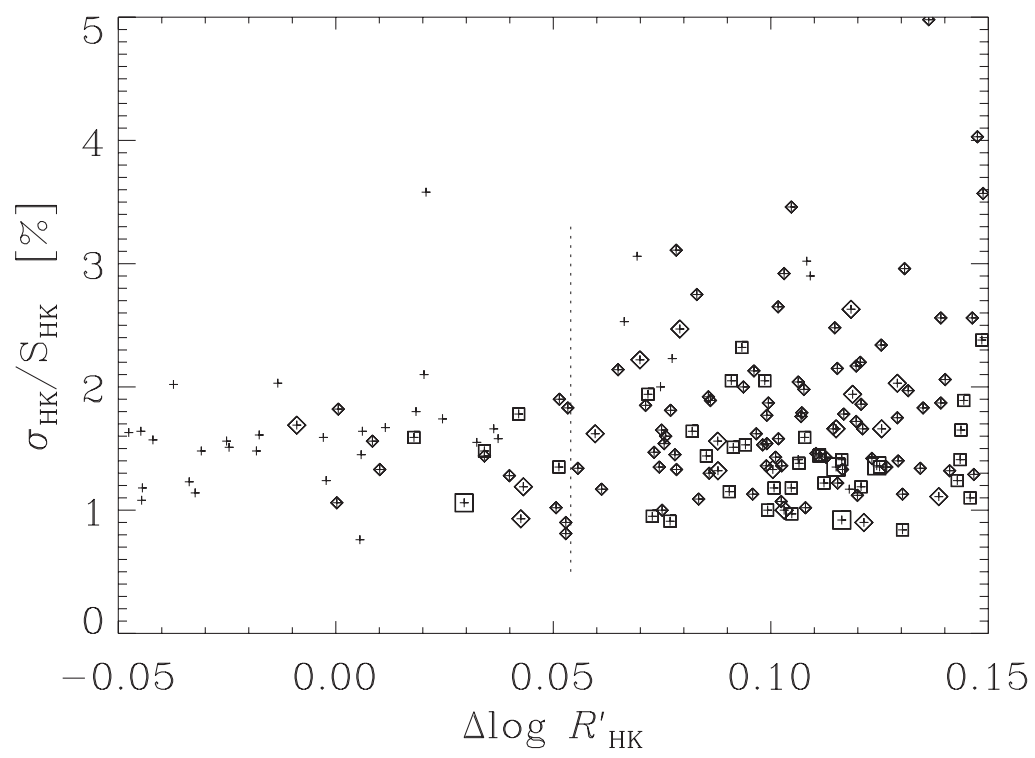

Figure 3. Fractional raw $S_{\mathrm{HK}}$ variability from Valenti \& Fischer (2005) vs. distance $\Delta \log R_{\mathrm{HK}}^{\prime}=\log R_{\mathrm{HK}}^{\prime}-\log R_{\mathrm{HK}}^{\prime}(\min )$ from dwarf $R_{\mathrm{HK}}^{\prime}(\min )$ boundary in Fig. 2 for stars with $t_{\mathrm{obs}}>4$ years; non-dwarfs are + , dwarfs with $t_{\mathrm{obs}}>4,5,6$, or 7 years of data are enclosed by small diamonds and small squares, large diamonds and large squares, respectively. Below $\Delta \log R_{\mathrm{HK}}^{\prime}=0.054$ (dotted), all dwarfs show $\sigma_{S} / S_{\mathrm{HK}}<2 \%$; above this, maximum variability rapidly increases. We propose dwarfs with $\Delta R_{\mathrm{HK}}^{\prime} \leqslant 0.054$ and data spanning $t_{\mathrm{obs}}>4$ years are candidate magnetic grand minimum stars (MGM stars; from Saar 2011).

have a range of properties themselves; we can probably expect the same from their stellar analogs.

\section{Properties of the new set of MGM candidates}

The set of MGM candidates defined above can now be explored to determine their important properties (see also Saar 2011). Overall, $\approx 7 \%$ of the joint Wright et al. Valenti \& Fischer sample are MGM candidates. The fraction of candidates peaks near the solar $T_{\text {eff }}$ at $\approx 17 \%$, cuts off sharply for $T_{\text {eff }} \geqslant 6050 \mathrm{~K}(\sim \mathrm{F} 9)$, and averages $<7 \%$ elsewhere (Fig. 4). The distribution with $[\mathrm{M} / \mathrm{H}]$ is fairly even, with perhaps an increase at $[\mathrm{M} / \mathrm{H}]<-0.65$, though statistics are poor. A $T_{\text {eff }}-[\mathrm{M} / \mathrm{H}]$ correlation suggests a relatively small range of convective zone depths are allowed (Fig. 5). The MGM candidate $v \sin i$ distribution is not statistically different from that of stars just above the $\Delta R_{\mathrm{HK}}^{\prime}$ cutoff $(0.054<\delta \leqslant 0.108 ;$ Saar 2011).

These selection criteria may be overstrict, as they have been set conservatively to avoid false positives. For example, there may be MGM candidates among the low $\sigma_{\mathrm{HK}}$ stars with $\Delta R_{\mathrm{HK}}^{\prime}>0.054$. The criteria certainly exclude some stars which are otherwise good candidates. For example, $51 \mathrm{Peg}$, a low $\sigma_{\mathrm{HK}}$ star (Baliunas et al. 1995) with low X-ray emission (Poppenhäger et al. 2009) is excluded here only because $t_{\mathrm{obs}}<1$ year in the Wright et al. (2004) data set.

The MGM candidates form a fairly distinct group at the bottom of the $\Delta \log R_{\mathrm{HK}}^{\prime}$ distribution (Fig. 6), well separated from the (on average) more variable stars with 


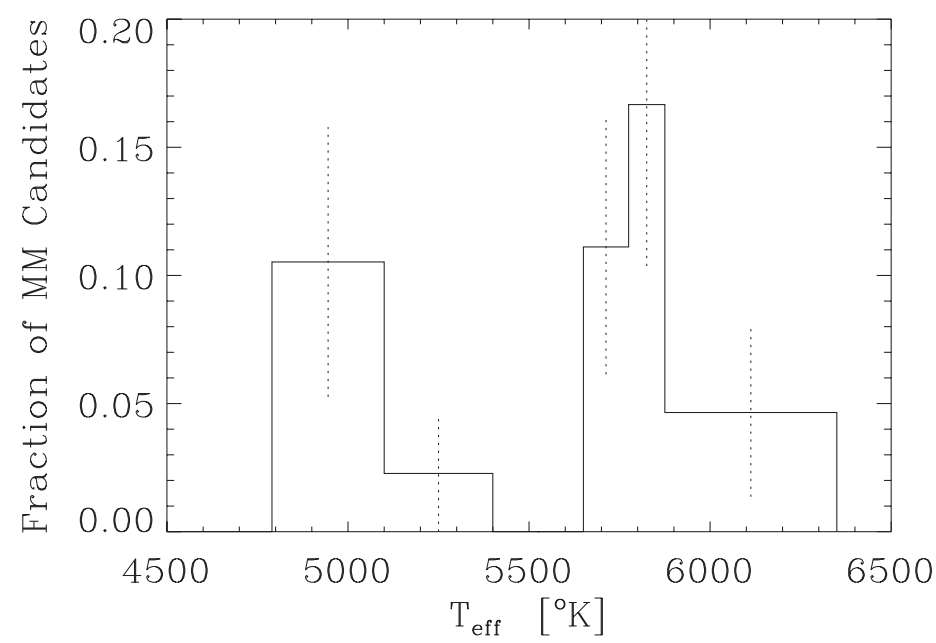

Figure 4. Distribution of MGM candidates observed for a duration $t_{\mathrm{obs}}>4$ years (as a fraction of all dwarfs in the sample with $t_{\mathrm{obs}}>4$ years) as a function of $T_{\text {eff }}$ with $\approx 41$ stars per bin. There is a peak at $T_{\text {eff }}=5825 \pm 50 \mathrm{~K}$, with no candidates showing $T_{\text {eff }}>6100$ (from Saar 2011).

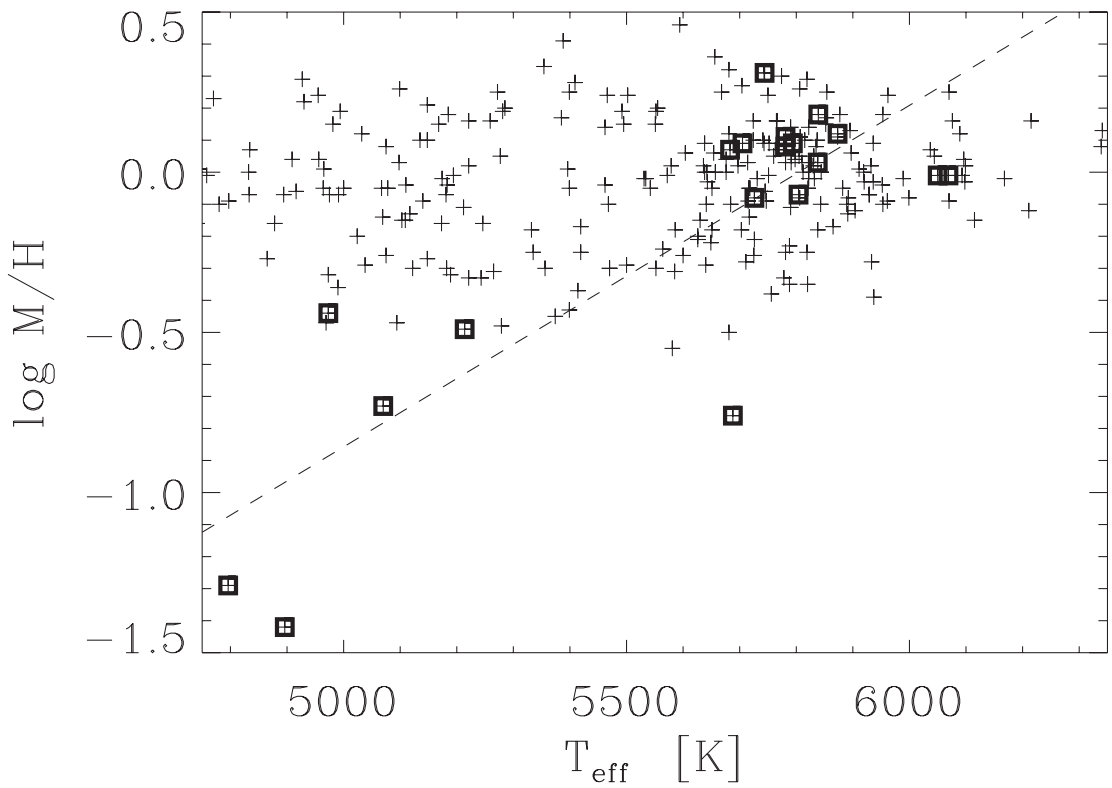

Figure 5. $\log \mathrm{M} / \mathrm{H}$ vs. $T_{\mathrm{eff}}$, for all dwarfs with $t_{\mathrm{obs}}>4$ years and for MGM candidates $t_{\mathrm{obs}}>4$ years (boxed). Unlike the overall sample, the MGM candidates show a distinct trend (dashed) of increasing metallicity with $T_{\text {eff }}$ (from Saar 2011).

$\Delta \log R_{\mathrm{HK}}^{\prime}$ values just above. This slight bimodal character brings back the possibility that the MGM is truly a distinct mode of the dynamo, rather than the tail of the distribution of behavior. The metallicity adjustment provided by using $\Delta \log R_{\mathrm{HK}}^{\prime}$ allows this bimodality to be seen; it is obscured without this correction. The gap is quite small though, and requires accurate $R_{\mathrm{HK}}^{\prime}$ data to discern. 


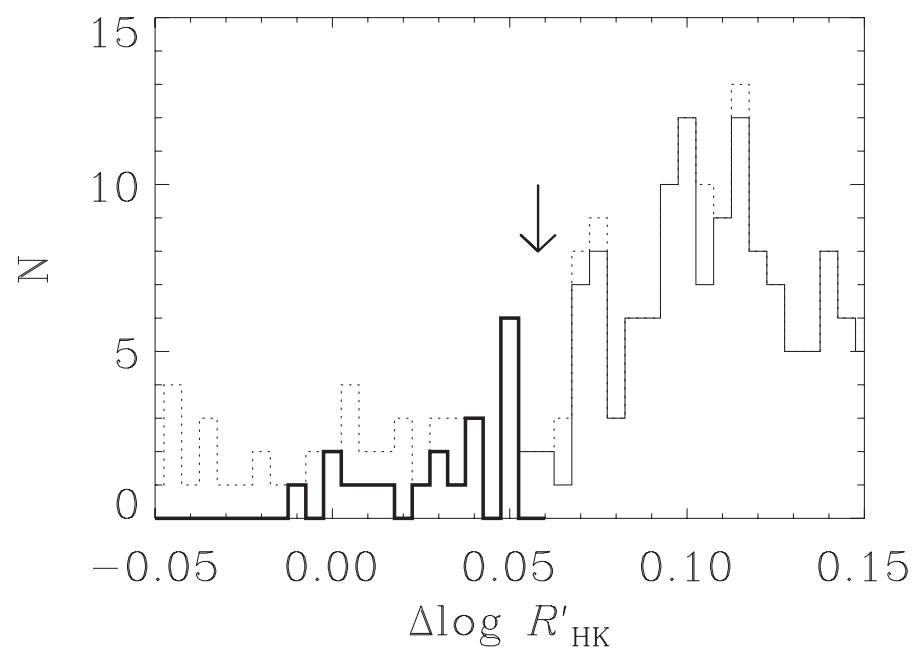

Figure 6. Histogram of $\Delta \log R_{\mathrm{HK}}^{\prime}$ for all stars (dotted), all dwarfs (solid), and MGM candidates defined in Fig. 3 (thick solid); $t_{\mathrm{obs}}>4$ years throughout. The MGM candidates form a fairly distinct population below a gap (arrow; from Saar 2011).

We have recently begun a study of the coronae of selected MGM candidates as defined by the above criteria ( $\S 3)$. X-rays are unambiguous markers of magnetic fields in non-accreting cool stars, since they are impossible to produce in any quantity without magnetic-driven heating. We used Chandra's ACIS-S to measure four MGM candidates, and one test case (matching the MGM criteria except for a too-short $t_{\text {obs }}$ ). We are still awaiting the data from one of these targets, but can give a preliminary report on the others here. Counts in the energy range $0.1-5 \mathrm{keV}$ were extracted within a 8-pixel radius circle and compared to a background in an annulus 16 to 30 pixels distant, centered on the apparent source position. A modification of this was needed for one source (HD 179958), whose common proper motion companion (HD 179957) was serendipitously detected 8" away. Here, wedges were removed from the background annuli of each, and we estimated the (small) cross-contamination of the two stars.

An accurate X-ray surface flux $F_{X}$ requires an good estimate of the mean coronal temperature $T_{X}$. Therefore, we also extracted counts for our sources between $0.2-5 \mathrm{keV}$, and used APEC models to predict how the count ratio $C_{0.1-5} / C_{0.2-5}$ would vary with $T_{X}$. We compute the X-ray luminosity $L_{X}$ using the best estimate $T_{X}$ (which were generally uncertain), Hipparcos distances $d$, and column density $N_{H}=0.07 \mathrm{~d} \mathrm{~cm}^{-2}$ with $d$ in parsecs. Our preliminary results and comparison stars are gathered in Table 1 . In all cases, the coronal temperatures for the MGM candidates were $T_{X} \leqslant 1 \mathrm{MK}$, indicating very cool coronae, similar to $51 \mathrm{Peg}$. The corresponding $F_{X}$ (computed using radii from Valenti \& Fischer 2005) were in good agreement with Schmitt (1997) and Schmitt \& Liefke (2004), with all the new MGM candidates having surface fluxes $F_{X} \sim 10^{4}$ ergs $\mathrm{cm}^{-2} \mathrm{~s}^{-1}$ (Fig. 7). The common proper motion (and thus presumably coeval) companion to candidate HD 179958 showed about $\sim 3$ times higher $F_{X}$ and a slightly warmer $T_{X}$, demonstrating a notable coronal activity difference at fixed age and similar mass.

The one exceptional star showing $F_{X} \ll 10^{4} \mathrm{ergs}_{\mathrm{cm}}^{-2} \mathrm{~s}^{-1}$, HD 157214, was also a test case MGM candidate with $t_{\mathrm{obs}}<1$ year. Indeed, Hall et al. (2009) observed the star recently showing notably higher $R_{\mathrm{HK}}^{\prime}$, suggesting Wright et al. (2004) observed the star is a temporary (cycle?) minimum. In this respect, the star may be similar to $\alpha$ Cen $\mathrm{A}$, 
Table 1. Coronae of MGM Candidates and Comparison Stars

\begin{tabular}{lccccccccccc}
\hline HD & $\mathrm{V}$ & $\begin{array}{c}\text { Spec. } \\
\text { type }\end{array}$ & $\begin{array}{c}T_{\text {eff }}^{1}[\mathrm{~K}] \\
{[\mathrm{K} / \mathrm{H}]^{1}}\end{array}$ & & $\log g^{1}$ & $\log R_{\mathrm{HK}}^{\prime}{ }^{2}$ & $\begin{array}{c}\sigma_{S}^{2} \\
{[\%]}\end{array}$ & $\begin{array}{c}t_{\text {obs }}^{2} \\
{[\mathrm{yr}]}\end{array}$ & $\begin{array}{c}L_{X} / 10^{27} \\
{\left[\mathrm{ergs} \mathrm{s}^{-1}\right]}\end{array}$ & $\log T_{X}$ & notes \\
\hline 50806 & 6.04 & G5V & 5685 & 0.07 & 4.36 & -5.10 & 1.28 & 6.4 & 2.8 & $5.8:$ & new \\
120066 & 6.30 & G0,5IV-V & 5873 & 0.12 & 4.23 & -5.15 & 1.82 & 6.4 & 1.9 & $5.9:$ & new \\
157214 & 5.40 & G0V & 5695 & -0.15 & 4.50 & -5.04 & 0.99 & 0.2 & 0.2 & $5.8:$ & new \\
179958 & 6.57 & G4V & 5760 & 0.05 & 4.39 & -5.08 & 1.34 & 6.3 & 2.0 & $5.8:$ & new \\
10700 & 3.50 & G8V & 5283 & -0.36 & 4.59 & -4.98 & 1.35 & 3.0 & 0.5 & $6.0:$ & $\tau$ Cet \\
207014 & 5.49 & G5V & 5787 & 0.15 & 4.45 & -5.08 & $\ldots$ & 0.2 & $0.63^{3}$ & $<6.0$ & 51 Peg \\
128620 & -0.01 & G2V & 5801 & 0.19 & 4.33 & $\ldots$ & $\ldots$ & $\ldots$ & $0.20^{4}$ & $\leqslant 6.0$ & $\alpha$ Cen A, in min. \\
179957 & 6.75 & G6V? & 5676 & 0.00 & 4.34 & -5.05 & 1.0 & 6.3 & 2.4 & $5.9:$ & new, non-MGM \\
\hline
\end{tabular}

Notes: ${ }^{1}$ from Valenti \& Fischer $(2005) ;{ }^{2}$ from Wright et al. (2004); ${ }^{3}$ from Poppenhäger et al. $(2009) ;{ }^{4}$ from Ayres et al. (2008)

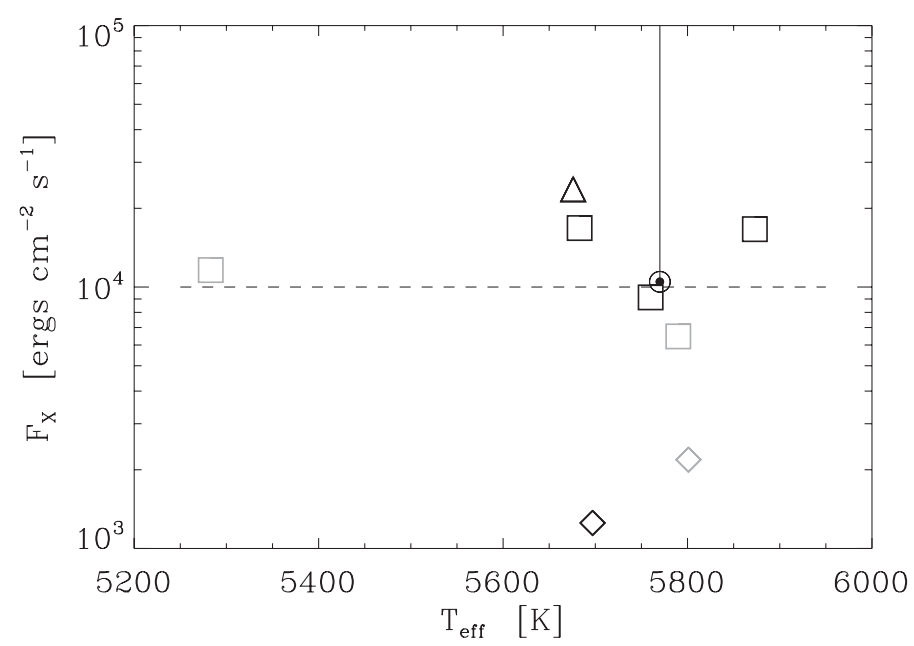

Figure 7. $T_{\text {eff }}$ vs. X-ray surface flux $F_{X}$ for the stars in Table 1. Squares are MGM candidates, diamonds are stars in temporary (cycle?) minima, the triangle is the non-MGM companion of HD 179958, and gray symbols are from the literature. The Sun at cycle minimum is marked $(\odot)$ as is the minimum $F_{X}$ level in dwarfs seen by Schmitt (1997) (dashed).

which recently became extremely faint and cool in X-rays for a period of a few years (Ayres et al. 2008).

\section{Discussion}

The MGM candidate selection method above can be refined to better remove the residual metallicity effects. Recall that $R_{\mathrm{HK}}^{\prime}=\left(F_{\mathrm{HK}}-F_{\mathrm{phot}}\right) / F_{\mathrm{bol}}$ (Noyes et al. 1984). The correction used above (Saar 2011) is a bulk adjustment to $R_{\mathrm{HK}}^{\prime}$, not considering the (possibly different!) individual contributions of $F_{\mathrm{HK}}(\mathrm{M} / \mathrm{H}), F_{\mathrm{phot}}(\mathrm{M} / \mathrm{H})$ and $F_{\mathrm{bol}}(\mathrm{M} / \mathrm{H})$. Since $T_{\text {eff }}(\mathrm{M} / \mathrm{H})$ is known for these stars from Valenti \& Fischer $(2005), F_{\text {bol }}$ can be corrected for separately. If both $F_{\mathrm{HK}}$ and $F_{\text {phot }}$ scale similarly with M/H, then defining a minimum $R_{\mathrm{HK}}^{\prime}$ as a function $\mathrm{M} / \mathrm{H}$ with an $M / H$ corrected $F_{\text {bol }}$ should be a better estimate of the true minimum Ca II HK in dwarfs. An improved analysis along these lines is in progress (Saar 2012, in prep.). Ultimately though, a full recalibration of $R_{\mathrm{HK}}^{\prime}$ might be needed for optimum results. 
Radial velocity surveys for exoplanets tend to avoid more active stars, since they have higher levels of velocity "jitter" due to the rotation and evolution of active regions. The stellar sample here, drawn from such a survey, is thus biased towards optically brighter, lower activity stars. Effectively, this means that the sample includes few stars younger than $\sim 1$ Gyr, but should be representative of older nearby dwarfs.

The typical X-ray levels of the MGM candidates are similar to inactive areas of the Sun (Schmitt 1997) and the average Sun at cycle minimum (Judge et al. 2003). Dwarf stars can apparently occasionally drop below this level (HD 157214, $\alpha$ Cen A), but this may be only in brief excursions, in which their coronae are very cool $\left(T_{X} \ll 10^{6}\right.$ $\mathrm{K})$. Perhaps these stars become briefly dominated with coronal holes, the coolest solar coronal structures. In other magnetic activity diagnostics, MGM candidates would seem to be only marginally less active $\left(R_{\mathrm{HK}}^{\prime}\right)$ or similar to (FUV lines; Judge \& Saar 2007) the cycle minimum Sun. By implication, solar emission in MGM may not be much different from a deep cycle minimum. Barring actually seeing a star enter or leave an MGM then (e.g., perhaps Donahue et al. 1995), low activity variability over long timescales may be more indicative of MGM stars than just a low average level.

Comparing with Giampapa et al. (2006), the fraction in our sample in the roughly equivalent $T_{\text {eff }}$ range $\left(5700 \leqslant T_{\text {eff }} \leqslant 5830\right)$ to the near-solar sample $(0.63 \leqslant \mathrm{~B}-\mathrm{V} \leqslant 0.67)$ in M67 is $12.5 \%$ (7 out of 56 ). This would suggest that near-solar stars at 4 Gyr may have a higher rate of MGM behavior (19\%) than for our sample of mixed ages. However, the M67 sample includes some binaries. As binaries are not strictly solar-like, and even wide binaries may have their rotational evolution (and, thus, perhaps their activity and dynamos) altered relative to single stars (Meibom et al. 2006), it might be safer to exclude these stars from the comparison. If we remove binaries, the MGM fraction of the M67 sample there drops to $\approx 12 \%$ ( 2 of 17 ), very similar to our result. This might then be a better estimate of the amount of time the Sun should spend in MGM.

Long term study of older clusters (e.g., M67, NGC 752, Rup 147, Kepler clusters NGC 6819 and 6791) will help better define the dependence of the MGM phenomenon and its properties with age. Also needed are more X-ray and $P_{\text {rot }}$ measurements, though these will not be trivial. In particular, if the new MGM candidates are well chosen, they should show a $P_{\text {rot }}$ distribution similar to low activity cycling stars of the same mass. Comparing Rossby number distributions may be useful, (e.g., Saar 1998). How does MGM frequency change with stellar age and rotation? How tightly restricted is the MGM mass dependence? How does the spectrum of MGM durations vary with stellar parameters? Some progress has been made, but the present indications need refinement and there is still much left to learn!

\section{Acknowledgments}

This work was supported by a Solar Heliospheric Guest Investigator grant NNX10AF29G, and Chandra grant GO1-12036X. We are grateful to J. Wright, P. Judge, J. Schmitt, T. Ayres, and K. Poppenhäger for many helpful discussions.

\section{References}

Ayres, T. R., Judge, P. G., Saar, S. H., \& Schmitt, J. H. M. M. 2008, ApJ, 678, L121

Baliunas, S. L., Donahue, R. A., Soon, W. H., et al. 1995, ApJ, 438, 269

Baliunas, S. L. \& Vaughan, A. H. 1985, ARAA, 23, 379

Baliunas, S. \& Jastrow, R. 1990, Nature, 348, 520 
Bercik, D. J., Fisher, G. H., Johns-Krull, C. M., \& Abbett, W. P. 2005, ApJ, 631, 529

Donahue, R. A., Baliunas, S. L., Soon, W. H., \& McMillan, F. M. 1995, IAU Symposium, 176, $72 \mathrm{P}$

Giampapa, M. S., Hall, J. C., Radick, R. R., \& Baliunas, S. L. 2006, ApJ, 651, 444

Hall, J. C., Henry, G. W., Lockwood, G. W., Skiff, B. A., \& Saar, S. H. 2009, AJ, 138, 312

Hall, J. C. \& Lockwood, G. W. 2004, ApJ, 614, 942

Henry, T. J., Soderblom, D. R., Donahue, R. A., \& Baliunas, S. L. 1996, AJ, 111, 439

Jenkins, J. S., Jones, H. R. A., Pavlenko, Y., et al. 2008, A\& A, 485, 571

Judge, P. G. \& Saar, S. H. 2007, ApJ, 663, 643

Judge, P. G., Saar, S. H., Carlsson, M., \& Ayres, T. R. 2004, ApJ, 609, 392

Judge, P. G., Solomon, S. C., \& Ayres, T. R. 2003, ApJ, 593, 534

Meibom, S., Mathieu, R. D., \& Stassun, K. G. 2006, ApJ, 653, 621

Noyes, R. W., Hartmann, L. W., Baliunas, S. L., Duncan, D. K., \& Vaughan, A. H. 1984, ApJ, 279,763

Pace, G. \& Pasquini, L. 2004, A\&A, 426, 1021

Poppenhäger, K., Robrade, J., Schmitt, J. H. M. M., \& Hall, J. C. 2009, A\& A, 508, 1417

Saar, S. H. 1998, Cool Stars, Stellar Systems, and the Sun 10, ASP Conf. Ser. Vol. 154, 211

Saar, S. H. \& Baliunas, S. L. 1992, The Solar Cycle, ASP Conf. Ser. Vol. 27, 150

Saar, S. H. 2006, Bulletin of the American Astronomical Society, 38, 240

Saar, S. H. 2011, Cool Stars, Stellar Systems, and the Sun 16, online poster volume.

Saffe, C., Gómez, M., \& Chavero, C. 2005, A\& A, 443, 609

Schmitt, J. H. M. M. 1997, A\&SA, 318, 215

Schmitt, J. H. M. M. \& Liefke, C. 2004, A\&SA, 417, 651

Usoskin, I. G., Mursula, K., Solanki, S., Schüssler, M., \& Alanko, K. 2004, A\&̋A, 413, 745

Valenti, J. A. \& Fischer, D. A. 2005, ApJS, 159, 141

Wilson, O. C. $1978, A p J, 226,379$

Wright, J. T. 2004, AJ, 128, 1273

Wright, J. T., Marcy, G. W., Butler, R. P., \& Vogt, S. S. 2004, ApJS, 152, 261

\section{Discussion}

Volker Bothmer: If you had looked at the Sun for the last two or three years, you would have been in the band that you are considering as Maunder Minimum?

Steve SaAR: Not quite, at least in Calcium. Its still a little high - in the chromosphere at least, the Maunder Minimum candidates seem to be a little bit lower.

DiBYENDU NANDY: It seems to me that your candidates with spots all have different coronal temperatures and different X-ray fluxes and presumably different magnetic fluxes. Given this, is it fair to conclude that there is not a single, absolute for stellar minimum active regions?

STEVe SAAR: They are sort of scattered, but within the large uncertainties in the temperatures I think we can say that $10^{4}$ is a reasonable estimate within the error bars we currently have. It would be nice to have an X-ray mission that could look nice and soft, and get better values than these, but unfortunately such a mission doesn't exist.

RAMON LOPEZ: I didn't catch how many stars were actually observed? Given the amount of stars that you are observing, how long would it actually take you to actually catch a "smoking gun" of a star going into or coming out of a Maunder Minimum?

STEve SAAR: The number of stars in the survey was about 1200 , with about 600 being true dwarfs in the end. The calculation of how long to see a Maunder state is not quite 
so straightforward: F and K stars are mostly not so good, so only the G stars which are even a smaller subset of the sample that we actually see 13 .

JEFF LiNsKY: I barely heard the word "solar twin" - a comment: people should look systematically (for many years) at solar twins (stars very nearly like the Sun).

Steve SaAR: Agreed, but the number of really good "solar twins" is small. We need more of them too! 